\title{
Critical exponents of words over 3 letters
}

\author{
Elise Vaslet \\ Institut de Mathématiques de Luminy, Université Aix-Marseille II \\ Marseille, France \\ vaslet@iml.univ-mrs.fr
}

Submitted: Oct 5, 2010; Accepted: May 25, 2011; Published: Jun 6, 2011

Mathematics Subject Classification: 68R15

\begin{abstract}
For all $\alpha \geq R T(3)$ (where $R T(3)=7 / 4$ is the repetition threshold for the 3-letter alphabet), there exists an infinite word over 3 letters whose critical exponent is $\alpha$.
\end{abstract}

\section{Introduction}

Let $A$ be a finite alphabet. Any finite word $v$ over $A, v \neq \epsilon$, can be factorized as $v=p^{k} e$, where:

$-k \geq 1$

- $e$ is a prefix of $p$

- $|p|$ is minimal

We then say that $v$ has period $p$, excess $e$, and exponent $E(v)=|v| /|p|$. For example, the English word church has period chur, excess ch, and exponent 3/2, while the French word entente has period ent, excess $e$, and exponent 7/3. A (finite or infinite) word is said to be $\alpha$-free (resp. $\alpha^{+}$-free) if none of its subwords has exponent $\beta$, with $\beta \geq \alpha$ (resp. $\beta>\alpha)$.

The critical exponent of an infinite word $w$ over $A$ is defined as

$$
E_{c}(w)=\sup \{E(v) \in \mathbb{Q}, v \text { subword of } w\}
$$

For example, the binary word $a b a b^{2} \cdots a b^{n} \cdots$ has critical exponent $+\infty$. The Thue-Morse word, fixed point of the morphism $0 \mapsto 01,1 \mapsto 10$, has critical exponent 2 ([10] and [1]). The Fibonacci word, fixed point of the morphism $0 \mapsto 01,1 \mapsto 0$, has critical exponent $2+\phi$, where $\phi$ is the golden number [8].

The problem to determine if, for a given real number $\alpha>1$, there is an infinite word $w_{\alpha}$ with critical exponent $\alpha$, has been solved by Krieger and Shallit [7]. The number of 
letters they use to construct the word $w_{\alpha}$ grows very fast as $\alpha$ tends to 1 , and they left open the construction of $w_{\alpha}$ over an alphabet with a fixed size.

Let $k$ be a natural number, and let $A_{k}$ be the $k$-letter alphabet. The repetition threshold on $k$ letters is the real number (see [6] and [3] for more details)

$$
R T(k)=\inf \left\{E_{c}(w), w \in A_{k}^{\omega}\right\}
$$

Dejean [6] conjectured that

$$
R T(k)= \begin{cases}2 & \text { if } k=2 \\ 7 / 4 & \text { if } k=3 \\ 7 / 5 & \text { if } k=4 \\ k /(k-1) & \text { if } k>4\end{cases}
$$

and this conjecture is now proved (see [9] and [5]).

It is clear that if $\alpha<R T(k)$, no word over $A_{k}$ has critical exponent $\alpha$. Currie and Rampersad [4] proved the following result for a binary alphabet:

For each $\alpha \geq 2=R T(2)$, there is an infinite binary word with critical exponent $\alpha$.

And they conjectured:

Let $k \geq 2$. For each $\alpha \geq R T(k)$, there is an infinite word over $k$ letters with critical exponent $\alpha$.

We will prove that this is true for $k=3$. Let $A_{3}=\{a, b, c\}$ be the 3-letter alphabet. Dejean [6] proved that $R T(3)=7 / 4$. We immediately remark that if $\alpha \geq 2$, the result of Currie and Rampersad gives us a binary word, and then also a word over $A_{3}$, with critical exponent $\alpha$. That's why we only have to consider the case $7 / 4 \leq \alpha<2$. To demonstrate her result, Dejean considered the morphism $\mu$ (which we will call Dejean's morphism) defined as follows:

$$
\mu:\left\{\begin{array}{l}
a \mapsto a b c a c b c a b c \text { bac bca cba } \\
b \mapsto \pi(\mu(a))=b c a \text { bac abc a cba cab acb } \\
c \mapsto \pi^{2}(\mu(a))=c a b \text { cba bca } b \text { acb abc bac }
\end{array}\right.
$$

where $\pi$ is the permutation $\left(\begin{array}{lll}a & b & c\end{array}\right)$, and proved that its fixed point $\mu^{\infty}(a)$ has critical exponent $7 / 4$.

\section{Exponents and Dejean's morphism}

Dejean [6] noticed the existence of specific subwords in $\mu(a), \mu(b)$, and $\mu(c)$, which can be used to desubstitute $\mu$. She called them characteristic factors: 
Proposition 1. The words $f_{1}=a b c a c b c, f_{2}=c a b c b a c$ and $f_{3}=c b c a c b a$ only appear in $\mu\left(A_{3}^{*}\right)$ respectively as prefix, central factor, and suffix, of $\mu(a)$.

Similarly, $\pi\left(f_{1}\right), \pi\left(f_{2}\right), \pi\left(f_{3}\right)$ only appear as prefix, central factor, and suffix of $\mu(b)$, and $\pi^{2}\left(f_{1}\right), \pi^{2}\left(f_{2}\right), \pi^{2}\left(f_{3}\right)$ as prefix, central factor, and suffix of $\mu(c)$.

Definition 1. The words $f_{1}, f_{2}$, and $f_{3}$ (resp. $\pi\left(f_{1}\right), \pi\left(f_{2}\right), \pi\left(f_{3}\right)$; resp. $\pi^{2}\left(f_{1}\right), \pi^{2}\left(f_{2}\right)$, $\left.\pi^{2}\left(f_{3}\right)\right)$ are called characteristic factors of $\mu(a)$ (resp. $\mu(b)$; resp. $\left.\mu(c)\right)$.

We use these characteristic factors to prove the following desubstitution results for $\mu$.

Proposition 2. Let $w$ be a word over $A_{3}$, and $u$ a subword of $\mu(w)$. If $|u| \geq 18$, then, there exists a unique $x \in A_{3}$, a unique $y \in A_{3}$, and there exist some unique $s, v, p \in A_{3}^{*}$, such that $u=s \mu(v) p$, where $s \neq \epsilon$ is a suffix of $\mu(x)$, and $p \neq \epsilon$ is a prefix of $\mu(y)$.

Proof. As $|u| \geq 18, u$ has a characteristic factor as a subword. The result is then clear since $\mu$ is a 19-uniform morphism, and since $\forall x \in A_{3}, \mu(x)$ begins and ends with $x$.

Theorem 1. Let $w \in A_{3}^{*}$, and let $u$ be a subword of $\mu(w)$. Assume $u$ has period $p$, excess $e$ and exponent $7 / 4<|u| /|p|<2$. Then, $w$ has a subword $v$ of length $|v| \geq\lceil|u| / 19\rceil$, with period $q$ such that $|q|=\lceil|p| / 19\rceil$, and with exponent $E(v) \geq|u| /|p|$.

Proof. There are two cases: either $|e| \geq 18$, or $|e|<18$.

Suppose first that $|e| \geq 18$. Then, we also have $|u| \geq 18$. Then, by Proposition 2 ,

- There exist some unique $x_{u}, y_{u} \in A_{3}$, and some unique $m_{u}, s_{u}, p_{u} \in A_{3}^{*}, s_{u} \neq \epsilon$ suffix of $\mu\left(x_{u}\right), p_{u}$ prefix of $\mu\left(y_{u}\right)$, such that $u=s_{u} \mu\left(m_{u}\right) p_{u}$,

- There exist some unique $x_{e}, y_{e} \in A_{3}$, and some unique $m_{e}, s_{e}, p_{e} \in A_{3}^{*}, s_{e} \neq \epsilon$ suffix of $\mu\left(x_{e}\right), p_{e}$ prefix of $\mu\left(y_{e}\right)$, such that $e=s_{e} \mu\left(m_{e}\right) p_{e}$.

Let:

$$
\begin{aligned}
& f=x_{e} m_{e} y_{e} \\
& v=x_{u} m_{u} y_{u}
\end{aligned}
$$

As $e$ is a suffix of $u$, we have: $p_{u}=p_{e}, y_{u}=y_{e}$, and $\mu\left(x_{e}\right) \mu\left(m_{e}\right)$ is a suffix of $\mu\left(x_{u}\right) \mu\left(m_{u}\right)$, thus $x_{e} m_{e}$ is a suffix of $x_{u} m_{u}$. Moreover, $e$ is a prefix of $u$, so $s_{u}=s_{e}$, $x_{e}=x_{u}, \mu\left(m_{e}\right) \mu\left(y_{e}\right)$ is a prefix of $\mu\left(m_{u}\right) \mu\left(y_{u}\right)$, and $m_{e} y_{e}$ is a prefix of $m_{u} y_{u}$ (see the following figure).

Therefore, $f$ is a prefix and a suffix of $v$, and $v$ has excess $f(|f|$ is maximal, otherwise $|e|$ would not be maximal in $u$ ). Let us denote its period by $q$, i.e. $v=q f$.

It is clear that

$$
|v| \geq\left\lceil\frac{|u|}{19}\right\rceil
$$




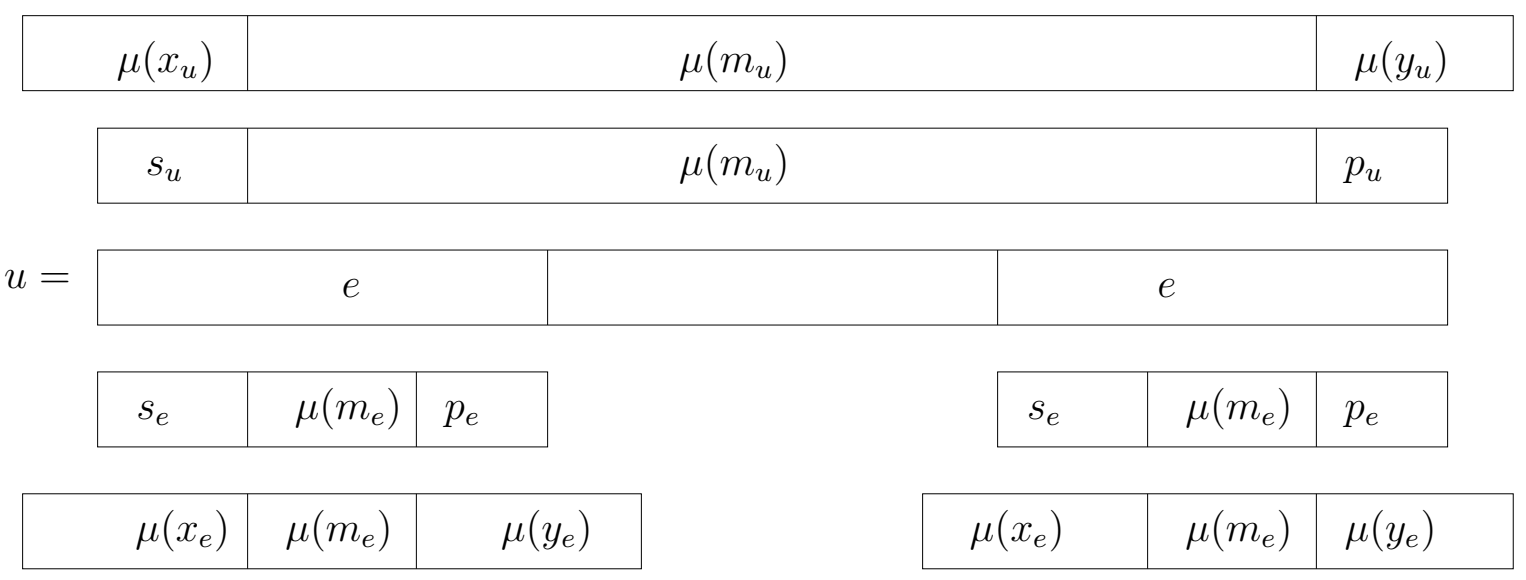

Moreover, $q$ has length

$$
\begin{aligned}
|q| & =|v|-|f|=\left|m_{u}\right|-\left|m_{e}\right| \\
& =\frac{\left|\mu\left(m_{u}\right)\right|-\left|\mu\left(m_{e}\right)\right|}{19} \\
& =\frac{\left(|u|-\left|s_{u}\right|-\left|p_{u}\right|\right)-\left(|e|-\left|s_{e}\right|-\left|p_{e}\right|\right)}{19} \\
& =\frac{|u|-|e|}{19}\left(\text { because } s_{u}=s_{e} \text { and } p_{u}=p_{e}\right) \\
& =\frac{|p|}{19}
\end{aligned}
$$

Finally, $v$ has exponent

$$
E(v)=\frac{|v|}{|q|} \geq\left\lceil\frac{|u|}{19}\right\rceil / \frac{|p|}{19} \geq \frac{|u|}{19} \cdot \frac{19}{|p|}=E(u)
$$

Thus, $v$ is the word we were looking for.

Suppose now that $|e|<18$. Then, as $E(u)>7 / 4,|u| \leq 24+18=42$. As $\mu$ is 19 uniform, $u$ is a subword of a word $\mu(x)$, where $x \in A_{3}^{*},|x| \leq 4$. Moreover, $E(u)>7 / 4$, so $x$ is not a subword of $\mu^{\infty}(a)$. Then, by looking at the $\mu(x)$ obtained if $x$ is not a subword of $\mu^{\infty}(a)$, we can again reduce the set of possible $x$ :

$$
x \in\{a a, b b, c c, a a a, b b b, c c c, a a a a, b b b b, c c c c, a b a b, a c a c, b a b a, b c b c, c a c a, c b c b\}
$$

since in the other cases, $\mu(x)$ has no subword $u$ such that $|u| \leq 42$ and $E(u)>7 / 4$. Then, we have:

- if $u$ is a subword of $\mu(x)$, with $x \in\{a a, b b, c c\}$, consider $v=x . v$ has exponent 2, and period $q$ of length $|q|=1$. We can also remark that $u$ necessarily has a period of length $19=19 \cdot|q|$, and has exponent $E(u) \leq 2=E(v)$. Therefore, $v$ is the word we were looking for. 
- if $u$ is a subword of $\mu(x)$, with $x \in\{a a a, b b b, c c c\}$, consider $v=x . v$ has exponent 3 , and period $q$ of length $|q|=1$. As $u$ necessarily has a period of length $19=19 \cdot|q|$, and has exponent $E(u) \leq 3=E(v), v$ is the word we were looking for.

- if $u$ is a subword of $\mu(x)$, with $x \in\{a a a a, b b b b, c c c c\}$, consider $v=x . v$ has exponent 4, and period $q$ of length $|q|=1$. As $u$ necessarily has a period of length $19=19 \cdot|q|$, and has exponent $E(u) \leq 4=E(v), v$ is the word we were looking for.

- finally, if $u$ is a subword of $\mu(x)$, with $x \in\{a b a b, a c a c, b a b a, b c b c, c a c a, c b c b\}$, consider $v=x . v$ has exponent 2 , and period $q$ of length $q=2$. As $u$ necessarily has a period of length $2.19=19 \cdot|q|$, and has exponent $E(u) \leq 2=E(v), v$ is the word we were looking for.

\section{Construction of an infinite $\alpha$-free word over $A_{3}$}

In the following, we will use the operator, denoted by $\delta$, that removes the first letter of a word: for example, $\delta(0110)=110$.

Lemma 1. Let $\mathfrak{L}$ be the set Fact $\left(\mu\left(A_{3}^{*}\right)\right)$ of all subwords of the words in $\mu\left(A_{3}^{*}\right)$. Let $\alpha \in] 7 / 4,2\left[\right.$ and $v \in A_{3}^{*}$, such that:

$-a b c b a b c v \in \mathfrak{L}$

- abcbabcv is $\alpha$-free.

Suppose that babcbabcv $=x u y$, where $u$ has exponent $E(u) \geq \alpha$. Then, $x=\epsilon$, and $u=b a b c b a b c$.

Proof. By hypothesis, abcbabcv is $\alpha$-free. Since $E(u) \geq \alpha, u$ is necessarily a prefix of babcbabcv. Moreover, babcbab has exponent $7 / 4<\alpha \leq E(u)$. Therefore, $u=b a b c b a b c v^{\prime}$, where $v^{\prime}$ is a prefix of $v$. Suppose that $v^{\prime} \neq \epsilon$.

By hypothesis, $a b c b a b c v^{\prime} \in \mathfrak{L}$. Moreover, the subword $a b c b a b c$ only appears in $\mathfrak{L}$ as a subword of $\mu(c)$. So $v^{\prime}=a b a c b a b c b a c \ldots$.

The excess of $u$ is at most $b a b c b a b$. Indeed, otherwise, the word $b a b c b a b c$, whose exponent is 2 , is a subword of $a b c b a b c v$, which is impossible since $a b c b a b c v$ is $\alpha$-free. Then, $u$ has excess $e$, with $|e| \leq 7$, and so has period $p$ with $|p| \leq 9$. So $u$ is a subword of babcbabcabacbabc. By looking at the factors of this word, we deduce that the only possibility is $u=b a b c b a b c$.

Lemma 2. Let $\alpha \in] 7 / 4,2\left[\right.$ be given. Let $s$, $t$ be natural numbers such that $\mu^{s}(b)=$ xabcbabcy, with $|x|=t$. Let $\beta=2-\frac{t}{4.19^{s}}$. Suppose that $7 / 4<\beta<\alpha$, and that abcbabcv $\in \mathfrak{L}$ is $\alpha$-free. Consider the word $w=\delta^{t} \mu^{s}$ (babcbabcv). Then, we have:

1. $w$ has a prefix with exponent $\beta$. 
2. If abcbabcv has a subword with exponent $\gamma$ and period $p$, then, w has a subword with exponent $\gamma$ and a period of length $19^{s}|p|$.

3. $w$ is $\alpha$-free.

Proof. $\quad$ 1. $\mu^{s}(b a b c b a b c)$ has exponent 2 and period $\mu^{s}(b a b c)$. We have $\left|\mu^{s}(b a b c)\right|=4.19^{s}$, and $\left|\mu^{s}(b a b c b a b c)\right|=8.19^{s}$, so the prefix $\delta^{t} \mu^{s}(b a b c b a b c)$ of $w$ has exponent

$$
\frac{|w|}{\left|\mu^{s}(b a b c)\right|}=\frac{\mid \mu^{s}(\text { babcbabc }) \mid-t}{\left|\mu^{s}(b a b c)\right|}=\beta
$$

2. Let $u$ be a subword of abcbabcv, with exponent $\gamma$ and period $p$. Then $\mu^{s}(u)$ is a subword of $\mu^{s}(a b c b a b c v)$, with exponent $\gamma$ and period $19^{s}|p|$. Moreover, $\mu^{s}$ (abcbabcv) is a suffix of $\delta^{t} \mu^{s}(b a b c b a b c v)$, since $t=|x| \leq\left|\mu^{s}(b)\right|$. So $\mu^{s}(u)$ is a subword of $w$, with the required properties.

3. Suppose that $w$ has a subword $u$, with exponent $\kappa \geq \alpha$ and period $p$. Then, by iteration of Theorem 2, babcbabcv has a subword $u^{\prime}$ with exponent $\kappa^{\prime} \geq \kappa$ and $q$ such that $|q|=\frac{|p|}{19^{s}}$. By Lemma 1 , as $\kappa^{\prime} \geq \alpha$, we deduce that $\kappa^{\prime}=2$ and that $u^{\prime}=b a b c b a b c$. Then $q=b a b c$, and $|p|=|q| \cdot 19^{s}=4.19^{s}$.

Moreover, $u$ is not a subword of $\mu^{s}(a b c b a b c v)$, otherwise $a b c b a b c v$ has a subword with exponent $\geq \alpha . u$ is not a subword of $\delta^{t} \mu^{s}(b a b c b a b c)$ either, otherwise, we would have $|u| \leq \mid \delta^{t} \mu^{s}($ babcbabc $) \mid=8.19^{s}-t$, and so:

$$
E(u)=\frac{|u|}{|p|} \leq \frac{8.19^{s}-t}{4.19^{s}}=\beta<\alpha,
$$

which is absurd since $E(u) \geq \alpha$.

Therefore, $u$ has a prefix $z$ such that $z=z_{1} \mu^{s}(a b c b a b c) z_{2}$, where $z_{1} \neq \epsilon$ is a suffix of $\mu^{s}(b)$, and $z_{2} \neq \epsilon$ is a prefix of $\mu^{s}(a)$ (since we remarked that the first letter of $v$ is a $a$ ). $z$ being a prefix of $u, z$ has a period of length $4.19^{s}$. Then, $z$ has period $z_{1} \mu^{s}(a b c) z_{1}^{\prime}$, with $z_{1}^{\prime}$ such that $\mu^{s}(b)=z_{1}^{\prime} z_{1}$. So we have:

$$
z=z_{1} \mu^{s}(a b c) z_{1}^{\prime} z_{1} \mu^{s}(a b c) z_{2} .
$$

We deduce that either $z_{2}$ is a prefix of $z_{1}^{\prime}$, or $z_{1}^{\prime}$ is a prefix of $z_{2}$. Yet neither is possible. Indeed, $z_{2}$ begins with the letter $a$, and $z_{1}^{\prime}$ begins with the letter $b$.

Finally, $w$ is $\alpha$-free.

\section{A word over $A_{3}$ with critical exponent $\alpha \geq R T(3)$}

Definition 2. A real number $\beta<\alpha$ is said to be obtainable if $\beta$ can be written as $\beta=2-\frac{t}{4.19^{s}}$, where the natural numbers $s$ and $t$ verify:

$-s \geq 3$ 
- the word $\delta^{t}\left(\mu^{s}(b)\right)$ begins with abcbabc.

We note that for any given $s \geq 3$, it is possible to choose $t$ such that

$-7 / 4<\beta=2-\frac{t}{4.19^{s}}<\alpha$

$-|\alpha-\beta| \leq \frac{19^{2}}{4.19^{s}}$

Indeed, $\mu^{2}(a), \mu^{2}(b)$, and $\mu^{2}(c)$ have length $19^{2}$, and each have $a b c b a b c$ as a subword. Therefore, choosing a large enough $s$, we can always find some obtainable real numbers $\beta$, arbitrarily close to $\alpha$.

Theorem 2. Let $\alpha \geq R T(3)=7 / 4$. Then, there is an infinite word over $A_{3}$ with critical exponent $\alpha$.

Proof. If $\alpha=7 / 4$, we already know that $\mu^{\infty}(a)$ has critical exponent $7 / 4$. If $\alpha \geq 2$, by the theorem for $k=2$, we can find a word over $A_{3}^{*}$ with critical exponent $\alpha$. Now, let $\alpha \in] 7 / 4,2[$.

Let $\left(\beta_{i}\right)_{i \in \mathbb{N}}$ be an increasing sequence of obtainable numbers, converging to $\alpha$. For each $i$, we write $\beta_{i}$ as:

$$
\beta_{i}=2-\frac{t_{i}}{4.19^{s_{i}}}
$$

where $s_{i}$ and $t_{i}$ are such that:

$-s_{i} \geq 3$

- $\delta^{t_{i}} \mu^{s_{i}}(b)$ begins with abcbabc.

For all words $v \in \mathfrak{L}$, let $\Phi_{i}(v)$ denote the word $\delta^{t_{i}} \mu^{s_{i}}(b v)$, and consider the following sequence:

$$
\begin{aligned}
v_{1} & =\Phi_{1}(a b c b a b c)=\delta^{t_{1}} \mu^{s_{1}}(b a b c b a b c) \\
v_{2} & =\Phi_{1} \Phi_{2}(a b c b a b c)=\delta^{t_{1}} \mu^{s_{1}}\left(b \delta^{t_{2}} \mu^{s_{2}}(\text { babcbabc })\right) \\
v_{3} & =\Phi_{1} \Phi_{2} \Phi_{3}(a b c b a b c) \\
\vdots & \\
v_{n} & =\Phi_{1} \Phi_{2} \Phi_{3} \ldots \Phi_{n}(a b c b a b c)
\end{aligned}
$$

By iteration of Lemma 2, as $a b c b a b c \in \mathfrak{L}$ is $\alpha$-free, we deduce that each $v_{i}$ is $\alpha$-free. Moreover, once again by Lemma 2 , each $v_{i}$ has a subword with exponent $\beta_{j}, j=1,2, \ldots, i$. Finally, consider the word $w=\lim _{n \rightarrow \infty} \in A_{3}^{\omega}$ (it is possible to take this limit since each $v_{i}$ is a prefix of $\left.v_{i+1}\right)$. $w$ then has critical exponent $\alpha$ : it is $\alpha$-free, yet has subwords with exponents $\beta_{i}$ converging to $\alpha$. 
The conjecture proposed by Currie and Rampersad in [4] is then true for alphabets of size 2 and 3. It still have to be proved for alphabets of size $\geq 4$. For that, another method must be found, because of Brandenburg's result in [2] : if $k \geq 4$, there is no $R T(k)$-free morphism, i.e., no morphism which maps, as Thue-Morse morphism for $k=2$ or Dejean's morphism for $k=3$, every $R T(k)$-free word to an $R T(k)$-free word.

\section{References}

[1] J. Berstel. Axel Thue's papers on repetitions in words: a translation. Publications du LaCIM, 20, 1995.

[2] F. J. Brandenburg. Uniformly growing $k$-th powerfree homomorphisms. Theoret. Comput. Sci., 23:69-82, 1983.

[3] A. Carpi. On Dejean's conjecture over large alphabets. Theoret. Comput. Sci., 385:137-151, 2007.

[4] J. D. Currie and N. Rampersad. For each $\alpha>2$ there is an infinite binary word with critical exponent $\alpha$. Electron. J. Combin., 15:N34, 2008.

[5] J. D. Currie and N. Rampersad. A proof of Dejean's conjecture. Preprint, http://arxiv.org/abs/0905.1129, 2009.

[6] F. Dejean. Sur un théorème de Thue. J. Comb. Theory A, 13:90-99, 1972.

[7] D. Krieger and J. Shallit. Every real number greater than 1 is a critical exponent. Theoret. Comput. Sci., 381:177-182, 2007.

[8] F. Mignosi and G. Pirillo. Repetitions in the Fibonacci infinite word. RAIRO Inform. Theor. Appl., 26:199-204, 1992.

[9] M. Rao. Last cases of Dejean's conjecture. WORDS 2009, http://www.labri.fr/perso/rao/publi/dejean.ps, 2009.

[10] A. Thue. Über die gegenseitige Lage gleicher Teile gewisser Zeichenreihen. Norske Vid. Selsk. Skr. Mat. Nat. Kl., 10:1-67, 1912. 